\title{
Di-Tyrosine Crosslinking and NOX4 Expression as Oxidative Pathological Markers in the Lungs of Patients with Idiopathic Pulmonary Fibrosis
}

\author{
Sanja Blaskovic ${ }^{1,2}{ }^{\oplus}$, Yves Donati ${ }^{1,2}$, Isabelle Ruchonnet-Metrailler ${ }^{1,2}{ }^{\oplus}$, Tamara Seredenina ${ }^{2}$, \\ Karl-Heinz Krause ${ }^{2}{ }^{(0)}$, Jean-Claude Pache ${ }^{3}$, Dan Adler ${ }^{4} \mathbb{D}$, Constance Barazzone-Argiroffo ${ }^{1,2, *}$ and \\ Vincent Jaquet ${ }^{2,5}$
}

1 Department of Pediatrics, Gynecology and Obstetrics, Children's Hospital, 1211 Geneva, Switzerland; Sanja.Blaskovic@unige.ch (S.B.); Yves.Donati@unige.ch (Y.D.); Isabelle.Ruchonnet-Metrailler@hcuge.ch (I.R.-M.)

2 Department of Pathology and Immunology, Medical School, University of Geneva, 1211 Geneva, Switzerland; tamara.seredenina@gmail.com (T.S.); Karl-Heinz.Krause@unige.ch (K.-H.K.); Vincent.Jaquet@unige.ch (V.J.)

3 Service of Clinical Pathology, Department of Pathology and Immunology, Medical School, University of Geneva, 1211 Geneva, Switzerland; Jean-Claude.Pache@hcuge.ch

4 Division of Pulmonary Diseases, Department of Medicine, Geneva University Hospitals, 1211 Geneva, Switzerland; Dan.Adler@hcuge.ch

5 READS Unit, Medical School, University of Geneva, 1211 Geneva, Switzerland

* Correspondence: Constance.Barazzone@hcuge.ch

check for updates

Citation: Blaskovic, S.; Donati, Y.; Ruchonnet-Metrailler, I.; Seredenina, T.; Krause, K.-H.; Pache, J.-C.; Adler, D.; Barazzone-Argiroffo, C.; Jaquet, V. Di-Tyrosine Crosslinking and NOX4 Expression as Oxidative Pathological Markers in the Lungs of Patients with Idiopathic Pulmonary Fibrosis. Antioxidants 2021, 10, 1833. https:// doi.org/10.3390/antiox10111833

Academic Editor: Stanley Omaye

Received: 18 October 2021

Accepted: 12 November 2021

Published: 18 November 2021

Publisher's Note: MDPI stays neutral with regard to jurisdictional claims in published maps and institutional affiliations.

Copyright: (c) 2021 by the authors. Licensee MDPI, Basel, Switzerland. This article is an open access article distributed under the terms and conditions of the Creative Commons Attribution (CC BY) license (https:// creativecommons.org/licenses/by/ $4.0 /)$.

\begin{abstract}
Idiopathic pulmonary fibrosis (IPF) is a noninflammatory progressive lung disease. Oxidative damage is a hallmark of IPF, but the sources and consequences of oxidant generation in the lungs are unclear. In this study, we addressed the link between the $\mathrm{H}_{2} \mathrm{O}_{2}$-generating enzyme NADPH oxidase 4 (NOX4) and di-tyrosine (DT), an oxidative post-translational modification in IPF lungs. We performed immunohistochemical staining for DT and NOX4 in pulmonary tissue from patients with IPF and controls using validated antibodies. In the healthy lung, DT showed little or no staining and NOX4 was mostly present in normal vascular endothelium. On the other hand, both markers were detected in several cell types in the IPF patients, including vascular smooth muscle cells and epithelium (bronchial cells and epithelial cells type II). The link between NOX4 and DT was addressed in human fibroblasts deficient for NOX4 activity (mutation in the CYBA gene). Induction of NOX4 by Transforming growth factor beta 1 (TGF $\beta 1$ ) in fibroblasts led to moderate DT staining after the addition of a heme-containing peroxidase in control cells but not in the fibroblasts deficient for NOX4 activity. Our data indicate that DT is a histological marker of IPF and that NOX4 can generate a sufficient amount of $\mathrm{H}_{2} \mathrm{O}_{2}$ for DT formation in vitro.
\end{abstract}

Keywords: idiopathic pulmonary fibrosis; NADPH oxidase; NOX4; di-tyrosine; immunohistochemistry; human lung tissue

\section{Introduction}

Idiopathic pulmonary fibrosis (IPF) is a progressive interstitial lung disease leading to lung tissue stiffness and fibrosis, decreased lung function and eventually respiratory failure. It is estimated that three million people are affected by IPF, worldwide. The median survival of IPF is estimated at 2-3 years after diagnosis [1,2]. IPF is characterized by the aberrant proliferation and activation of fibroblasts as well as the presence of myofibroblasts that secrete excessive collagen and extracellular matrix (ECM) leading to the scarring of lung tissue and destruction of the alveolar epithelium (reviewed in [3]). Lung remodeling is thought to occur following repetitive injuries leading to alveolar epithelial cells (AEC) apoptosis, proliferation of fibroblasts and Transforming growth factor beta 1 (TGF $\beta 1$ )-induced myofibroblast differentiation as well as endothelial dysfunction. However, knowledge of the underlying molecular mechanisms leading to IPF is scarce and the clinically approved 
antifibrotic agents Nintedanib and Pirfenidone provide only limited benefit and are associated with adverse effects. Characterization of novel alternative pathogenic pathways is strongly needed to improve our knowledge on this devastating disease and develop novel classes of therapeutics.

A key hallmark of IPF pathogenesis is the presence of high levels of reactive oxygen species (ROS) in the lungs (reviewed in [4]). Although, the sources of ROS can be multiple, NOX4 represents a key enzymatic source of ROS in IPF lungs and has been associated to fibrogenic properties in the lungs [5,6]. NOX4 is a member of the family of nicotinamide adenine dinucleotide phosphate (NADPH) oxidases, whose sole function is the generation of ROS [7]. NOX4 expression is strongly upregulated in fibroblasts from IPF patients and following treatment with the fibrogenic cytokine TGF $\beta 1$ leading to extracellular $\mathrm{H}_{2} \mathrm{O}_{2}$ production [5]. In addition, NOX4-deficient mice show decreased pulmonary fibrosis and AEC cell death in a mouse model of bleomycin-induced lung fibrosis [6]. NOX4 inhibitors are currently investigated in clinical trials for IPF (NCT03865927). Nevertheless, the precise molecular mechanisms by which NOX4-derived ROS contribute to IPF pathology are not known. ROS are often considered as harmful molecules directly damaging cellular components, but ROS (and in particular $\mathrm{H}_{2} \mathrm{O}_{2}$ ) also exert a physiological function as signaling molecules [8]. In fact, $\mathrm{H}_{2} \mathrm{O}_{2}$ can serve as a substrate for enzymatic reaction catalyzed by peroxidases in various biological reactions, such as the generation of hypochlorous acid $(\mathrm{HOCl})$ for germ killing by granulocytes and thyroid hormone synthesis [9]. One such specific reaction is called di-tyrosine (DT) crosslinking. In vitro, DT formation is catalyzed by a heme-containing peroxidase in the presence of $\mathrm{H}_{2} \mathrm{O}_{2}$ leading to the formation of a covalent carbon-carbon bond between two tyrosine residues of the same, or two adjacent proteins [10]. This phenomenon has physiological relevance and is well described in invertebrates. For instance, during egg fertilization of the sea urchin egg, the combined activity of a NADPH oxidase (Udx1) and a secreted ovoperoxidase is required for crosslinking of protein tyrosyl residues to create a stiffened ECM around the egg to avoid polyspermy [11]. However, DT is also present in different pathological conditions such as Alzheimer's disease [12], Parkinson's disease [13], atherosclerotic plaque formation [14], and IPF. A study by Pennathur and collaborators used mass spectrometric quantification of oxidative tyrosine modifications and documented a significant increase in the plasma of patients with interstitial lung disease and in the lungs of bleomycin-treated mice [15]. As of today, we are still unaware whether DT is formed in the lungs of IPF patients, and how its levels and localization contribute to IPF pathogenesis. A recent study has shown that the DT cross-linking of fibronectin, a key protein of the ECM, alters functional fibronectin characteristics in vitro [16]. In fact, ECM properties and glycogen crosslinking are known to be altered in IPF patients, thereby contributing to tissue stiffness [17]. In terms of mechanism, neither the $\mathrm{H}_{2} \mathrm{O}_{2}$ sources nor the potential peroxidases involved in DT formation in humans are known.

Thus, we aimed at addressing the localization and levels of DT as well as NOX4 as the potential source of $\mathrm{H}_{2} \mathrm{O}_{2}$ involved in DT generation in the lungs of patients with IPF. We showed that normal lungs were virtually devoid of DT while lung tissue from IPF patients were characterized by a significant DT staining. NOX4 was a sufficient source of $\mathrm{H}_{2} \mathrm{O}_{2}$ to form DT in vitro, but NOX4 expression in IPF tissue did not clearly correlate with DT localization, especially in the smooth muscle cells of IPF lungs. Altogether, we conclude that DT and NOX4 expression patterns are strongly affected in IPF while DT represents a key novel histopathological feature of IPF.

\section{Materials and Methods}

\subsection{Antibodies, Enzymes and Reagents}

The anti-di-tyrosine monoclonal antibody clone 1C3 was purchased from JaiCa, Fukuroi, Japan and the rabbit monoclonal antibody directed against the C-terminus of NOX4 was a kind gift of Prof Jansen Dürr, University of Innsbruck (Innsbruck, Austria). Isotype negative control antibodies were the following: mouse IgG2a (DAKO, X0943) for 
DT and recombinant rabbit IgG, monoclonal isotype control [EPR25A] (ACAM, ab172730) for NOX4. DAKO REAL Detection system peroxidase/DAB+, rabbit/mouse, K5001 was used for IHC on human tissues. Transforming Growth Factor- $\beta 1$ human was from Sigma (T7039, Saint Louis, MO 63103, USA). Alexa Fluor 488 tyramide Reagent (TSA) was from Life Technology, (B40953, Eugene, Oregon, USA). Fetal calf serum was purchased from Chemie Brunschwig (K007310G). Fluorescent secondary antibody for DT immunofluorescence was goat anti-mouse IgG DyLight594 (Jackson ImmunoResearch, Cambridge, UK, 115-515-003). Panexin basic was purchased from Pan Biotech (P04-96090, Aidenbach, Germany). Phosphate-Buffered Saline, PBS (14190, Life Technologies, Paisley, Scotland, UK), Hepes 1M (15630, Life Technologies, Paisley, Scotland, UK) and Hanks' Balanced Salt Solution (HBSS), calcium, magnesium, HBSS++ (14025, Life Technologies, Paisley, Scotland, UK) were from GIBCO. L-tyrosine was from Carl Roth (1741.1, Karlsruhe, Germany). Normal goat serum (NGS) was from Invitrogen (10000C, Waltham, Massachusetts, USA), DAPI from Roche Diagnostics (10236276001, Basel, Switzerland), FluorSave Reagent (345789) and $\mathrm{H}_{2} \mathrm{O}_{2}$ (107209) were from Merck (Merck KGaA, Darmstadt, Germany).

\subsection{Gene Expression in Fibroblasts}

The human lung fibroblast cell line MRC5 (ATCC CCL-171) and primary human skin fibroblasts isolated from a healthy donor (hSFs) and a patient carrying mutation in CYBA gene (hSFp22 deficient) [18], were maintained in Dulbecco's modified Eagle's medium (DMEM) supplemented with fetal bovine serum (FBS, 10\%), penicillin (100 U/mL), and streptomycin $(100 \mu \mathrm{g} / \mathrm{mL})$ at $37{ }^{\circ} \mathrm{C}$ in air with $5 \% \mathrm{CO}_{2}$. For differentiation experiments, the cells were kept in a serum-free medium for $24 \mathrm{~h}$ and treated with $2 \mathrm{ng}$ TGF- $\beta 1$ for $24 \mathrm{~h}$ and collected for RNA extraction.

RNA was extracted using RNeasy mini kit (Qiagen, Dusseldorf, Germany) according to manufacturer's protocol. Five hundred nanograms (500 ng) were used for cDNA synthesis using the PrimeScript RT reagent kit (Takara, Saint-Germain-en-Laye, France) following the manufacturer's instructions. Real-time PCR was performed using the SYBR green assay at the Genomics Platform, National Center of Competence in Research Frontiers in Genetics (Geneva, Switzerland), on a 7900HT SDS system (Applied Biosystems, Foster City, USA). The efficiency of each primer was assessed with serial dilutions of cDNA. Relative expression levels were calculated by normalization to the geometric mean of two housekeeping genes, $\beta 2$-microglobulin and GAPDH, as previously described [19]. Normalized quantities are reported as mean \pm SEM. The sequences of the primers used in this study are documented in Table 1.

Table 1. RT-qPCR primers used in the study.

\begin{tabular}{|c|c|c|c|}
\hline Gene & Accession Number & Primers Forward $5^{\prime}-3^{\prime}$ & Primers Reverse $5^{\prime}-3^{\prime}$ \\
\hline NOX4 & NM_001143836.3 & AACCGAACCAGCTCTCAGAA & TTGACCATTCGGATTTCCAT \\
\hline ACTA2 & NM_001141945.2 & AATACTCTGTCTGGATCGGTGGCT & ACGAGTCAGAGCTTTGGCTAGGAA \\
\hline FN1 & NM_001306129.2 & CGGTGGCTGTCAGTCAAAG & AAACCTCGGCTTCCTCCATAA \\
\hline COL1A1 & NM_000088.4 & GAGGGCCAAGACGAAGACATC & CAGATCACGTCATCGCACAAC \\
\hline GAPDH & NM_001256799.3 & GCACAAGAGGAAGAGAGAGACC & AGGGGAGATTCAGTGTGGTG \\
\hline$B 2 M$ & NM_004048.4 & TGCTCGCGCTACTCTCTCTTT & TCTGCTGGATGACGTGAGTAAAC \\
\hline
\end{tabular}

\subsection{TSA Assay and DT Immunofluorescence}

Fibroblasts were cultured on glass coverslips as described above. A starvation step was performed by adding new media with low FCS concentration ( $0.01 \%$ for HSF cells) or culture media supplemented with $10 \%$ panexin, and $0.1 \%$ FCS for MRC5 cells for $24 \mathrm{~h}$. TGF $\beta 1$ was added in the starvation media $(5 \mathrm{ng} / \mathrm{mL})$ for 7 days with medium change every 2-3 days. On the day of the experiment a $100 \times$ dilution of TSA was added with or without HRP $(5 \mathrm{U} / \mathrm{mL})$ and with or without $\mathrm{H}_{2} \mathrm{O}_{2}(10 \mu \mathrm{M})$ in TRIO buffer $(1 \mathrm{mM}$ Hepes, $1 \mathrm{mM}$ L-Tyrosine in HBSS++) for $1 \mathrm{~h}$ at RT. Cells were washed $2 \times$ with HBSS ++ and fixed with $4 \%$ paraformaldehyde for $15 \mathrm{~min}$ at room temperature (RT) and washed with PBS. 
Cells were blocked for $1 \mathrm{~h}$ at RT in blocking solution (PBS, 5\% NGS, 0.3\% TritonX-100). Cells were incubated with primary di-tyrosine antibody (dilution 1:400) at RT. After $1 \mathrm{~h}$, cells were washed 3 times for 5 min with PBS at RT and a secondary anti-mouse antibody conjugated to D594 was added for $1 \mathrm{~h}$ (dilution 1:200). Cells were again washed 3 times for $5 \mathrm{~min}$ at RT and cell nuclei were stained with DAPI $(1 \mu \mathrm{g} / \mathrm{mL}$ in PBS) $5 \mathrm{~min}$ at RT. Coverslips with cells were mounted with FluorSave and imaged with Axiocam Fluo (Carl Zeiss Microscopy GmbH, Jena, Germany).

\subsection{Human Lung Samples}

Human lung tissue samples used in this study (Table 2) were obtained from the department of Pathology, University Geneva hospital. Samples were collected either on biopsies or on necropsies. Clinical description was limited to definitive diagnosis made by the pathologist, the gender and the age of the patient. Samples were irreversibly anonymized, dated, processed and analyzed according to the Swiss Medical ethical guidelines and recommendations (Senate of the Swiss Academy of Medical Sciences, Basel, Switzerland, 23 May 2006). The study was approved by the regional research committee of the University Geneva Hospital (NAC10-052R; NAC 11-027R).

Table 2. Additional information of human lung tissue samples used in the study.

\begin{tabular}{|c|c|c|c|c|c|}
\hline Patient Number & Pathology & Age at Diagnosis & Gender & Origin of Sample & Lung Region \\
\hline 1 & IPF & 77 & M & Surgical biopsy & Right upper lobe \\
\hline 2 & IPF (fibrotic hypersensitivity pneumoniae) & 69 & $\mathrm{M}$ & Surgical biopsy & Right upper lobe \\
\hline 3 & IPF & 74 & $\mathrm{M}$ & Surgical biopsy & Left lower lobe \\
\hline 4 & IPF & 71 & $\mathrm{M}$ & Surgical biopsy & Left lower lobe \\
\hline 5 & IPF (pleural fibrosis) & 75 & $\mathrm{~N} / \mathrm{A}$ & Surgical biopsy & $\mathrm{N} / \mathrm{A}$ \\
\hline 6 & Papillary adenocarcinoma & 62 & $\mathrm{M}$ & Biopsy, adjacent tissue & Right middle lobe \\
\hline 7 & Undifferentiated lung adenocarcinoma & 69 & M & Biopsy, adjacent tissue & Left upper lobe \\
\hline 8 & Metastases from endometrial adenocarcinoma & 56 & $\mathrm{~F}$ & Biopsy, adjacent tissue & Left lower lobe \\
\hline 9 & Bullous emphysema & 30 & M & Biopsy, adjacent tissue & Right upper lobe \\
\hline 10 & Acute bronchitis & 85 & M & Postmortem material & N/A \\
\hline
\end{tabular}

IPF: idiopathic pulmonary fibrosis; M: male; F: female; N/A: not available.

\subsection{Immunohistochemical Staining}

Paraffin-embedded sections $(5 \mu \mathrm{m})$ of human control and IPF affected lungs were stained according to the cell signaling technology standard protocol [20] using DAKO Envision kit (K4011, Agilent Technologies, CA, USA) using DT and NOX4 antibodies.

Briefly, paraffin-embedded samples were deparaffinized using xylene and 95-100\% ethanol and subsequently hydrated in $\mathrm{H}_{2} \mathrm{O}$.

NOX4 labeling required pressure and heat-induced epitope retrieval (20 bar) in TrisEDTA, pH 9.0 (10 mM/1 mM) buffer. DT did not require antigen retrieval. Endogenous peroxidases were blocked with DAKO peroxidase block solution. Both primary and secondary antibodies were diluted with DAKO antibody diluent. The anti-DT mouse monoclonal antibody and its mouse IgG2a isotype control were used at $0.25 \mu \mathrm{g} / \mathrm{mL}$. The anti-NOX4 rabbit monoclonal antibody and its rabbit monoclonal Ig isotype control were used at $2.5 \mu \mathrm{g} / \mathrm{mL}$ ). We applied the primary antibodies for $1 \mathrm{~h}$ at RT. Finally, labeled polymer-horseradish peroxidase (HRP) anti-rabbit or anti-mouse (DAKO Envision system, Agilent Technologies, CA, USA) was used for $30 \mathrm{~min}$ at RT and the signal was visualized with diaminobenzidine (Envision system, Dako SA, Geneva, Switzerland). Sections were counterstained with hematoxylin (BioGnost, Zagreb, Croatia). Images were acquired using Axioscan Z1 microscope (Carl Zeiss Microscopy GmbH, Jena, Germany) and analyzed using the Definiens Developer XD ${ }^{\mathrm{TM}} 2.7$ software (Definiens AG, Munich, Germany). Quantification of DT and NOX4 was done in ImageJ (version 1.51, NIH, Rockville Pike, Bethesda, Maryland) on randomly selected 10 images/sample and statistical analysis was carried out with GraphPad PRISM (Prism 8.0.2, GraphPad Software, San Diego, CA, USA) using the Mann-Whitney nonparametric test. At least 10 adjacent sections were stained 
for each antibody. Control tissue consisted of adjacent healthy tissue for patients nos. 6-9 and from postmortem tissue for patient no. 10.

\section{Results}

Our study aimed at evaluating the pattern of expression of the $\mathrm{H}_{2} \mathrm{O}_{2}$-generating enzyme NOX4 and the presence of oxidative post-translational modification DT in human lungs. The NOX4 antibody used in this study was a rabbit monoclonal antibody directed against the intracellular $\mathrm{C}$ terminus of human NOX4 produced in the laboratory of Professor Pidder Jansen-Dürr, Institute for Biomedical Ageing Research. University of Innsbruck. This antibody belongs to the small list of available validated NOX4 antibodies, suitable for Western blot and IHC [21]. We previously documented the specificity of this antibody over other NOX isoforms [22] and its specificity for immunohistochemical detection in human tissue samples [23]. The mouse monoclonal DT antibody was generated using a DT conjugate as immunogen and selected for DT specificity [24]. In order to address the specificity of the DT antibody and the biological significance of NOX4 in DT formation, we used an in vitro fibroblast experimental system inspired by the methodology described by Larios et al. [25].

\subsection{Generation and Immunodetection of DT in Fibroblast Cell Culture}

We used MRC5 lung fibroblasts treated with TGF $\beta 1$ to induce myofibroblast differentiation as evidenced by increased expression of NOX4, alpha smooth muscle actin $(\alpha-S M A)$ and the ECM proteins fibronectin and collagen 1a [16] (Figure 1A). The hemecontaining peroxidase (HRP) and fluorescein-tyramide, a fluorescently labeled chemical analogue of tyrosine, were added to the fibroblast culture. Upon $\mathrm{H}_{2} \mathrm{O}_{2}$ addition, HRP converted fluorescein-tyramide into a highly-reactive tyramide radical that forms dimers with tyrosine residues allowing the detection of DT crosslinks formed with proteins of the ECM (Figure 1B). After washing unbound fluorescein tyramide, immunofluorescent staining using the anti-DT monoclonal antibody was performed. A striking colocalization of bound fluorescent tyramide and DT staining was observed (Figure 1B) confirming that the antibody recognized the DT formed between fluorescein tyramide and ECM proteins by the coordinated action of $\mathrm{H}_{2} \mathrm{O}_{2}$ and HRP.

\subsection{NOX4-Derived $\mathrm{H}_{2} \mathrm{O}_{2}$ Is Sufficient for DT Generation in Skin Fibroblasts}

In order to address whether NOX4 was able to generate a sufficient amount of $\mathrm{H}_{2} \mathrm{O}_{2}$ upon activation by TGF $\beta 1$ to generate DT, we used primary skin fibroblasts isolated from a patient with a mutation in $C Y B A$, the gene coding for p22phox [18], a necessary subunit for NOX4 activity [26]. Similar to MRC5, NOX4 was induced in both control and CYBAdeficient skin fibroblasts (Figure S1A). Interestingly, a weak overlapping staining was detected using both fluorescent tyramide and DT staining in the WT fibroblasts (Figure 1C, 1st and 2nd panel), which was completely absent in CYBA-deficient fibroblasts, whose NOX4 is not active (Figure 1C, 3rd panel). This suggests that NOX4 generates a sufficient amount of $\mathrm{H}_{2} \mathrm{O}_{2}$ for DT generation in differentiated skin myofibroblasts, provided that a heme containing peroxidase and tyrosine-rich proteins of the ECM are present.

\subsection{Global DT Staining Is Significantly Increased in the Lungs of IPF Patients}

In order to evaluate the presence of DT crosslinking and NOX4 expression in the lungs of IPF patients, we performed immunohistochemistry (IHC) for both markers. Isotype control antibodies were used to confirm the specificity of the staining (Figure S2). We detected high levels of DT in the lungs of IPF patients, indicative of a wide dissemination of ECM proteins and oxidative events. On the other hand, DT staining was barely above background in control tissue (Figure 2A,B). NOX4 staining was more localised than DT staining and NOX4 levels varied widely between individuals. Altogether, no significant change of global NOX4 levels was detected in the lungs of IPF patients compared to controls (Figure 2C,D). 
A

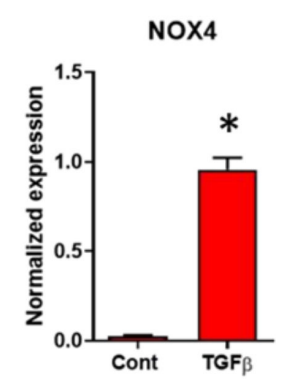

FN

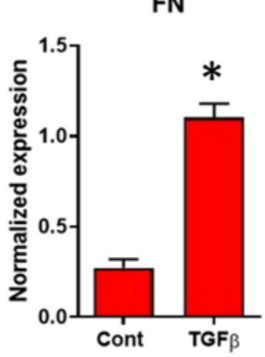

COL1A

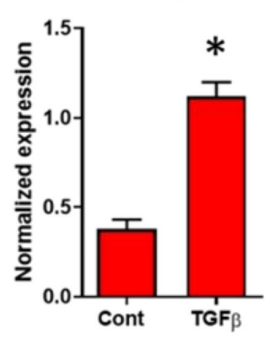

B DAPI TSA Di-TYR merge

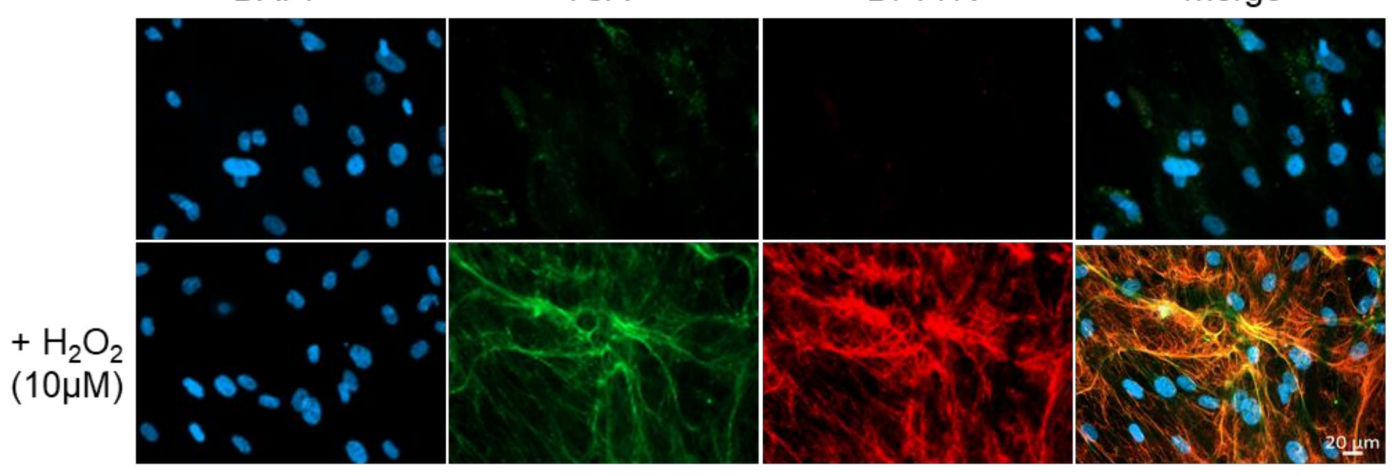

C

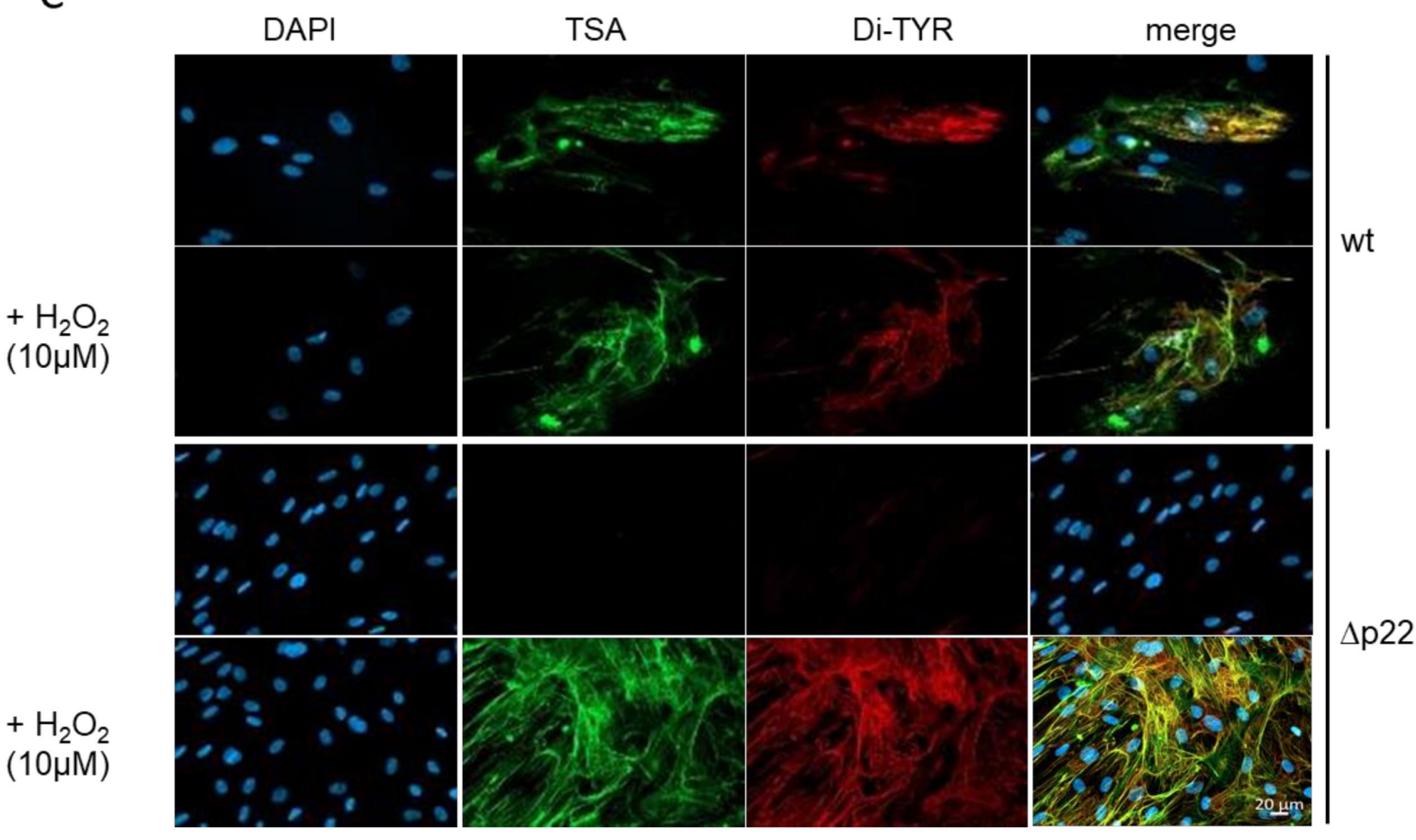

Figure 1. (A) TGF $\beta 1$-induced upregulation of the expression of NOX4, the myofibroblast marker alpha-SMA and proteins of the ECM in MRC5 human lung fibroblasts. $N=4,{ }^{*} p<0.05$ using Mann-Whitney nonparametric test. (B). Fluorescent microscopy of DT in TGF- $\beta 1$-treated MRC5. Addition of $\mathrm{H}_{2} \mathrm{O}_{2}$ in presence of HRP cells induces crosslinking of the fluorescein tyramide (TSA, green) with the extracellular matrix network. The cross-links are detected by the DT antibody (red) and colocalize with crosslinked TSA (yellow). (C). Similar experiment with human skin fibroblasts from a healthy donor and a patient carrying a p22 ${ }^{\text {phox }}$ mutation. Deletion of $\mathrm{p}^{22^{\text {phox }}}$ completely abolishes the formation of DT in the absence of exogenous $\mathrm{H}_{2} \mathrm{O}_{2}$. Blue, DAPI staining of cell nuclei. NOX4: NADPH oxidase isoform 4; ACTA 2: Actin Alpha2, Smooth Muscle; FN: Fibronectin; COL1A: Collagen1A; DAPI: 4',6-diamidino-2-phenylindole; TSA: Alexa Fluor 488 tyramide Reagent; Di-TYR: dityrosine; TGF $\beta$ : Transforming growth factor $\beta$; Cont: control; HRP: horseradish peroxidase. 
A

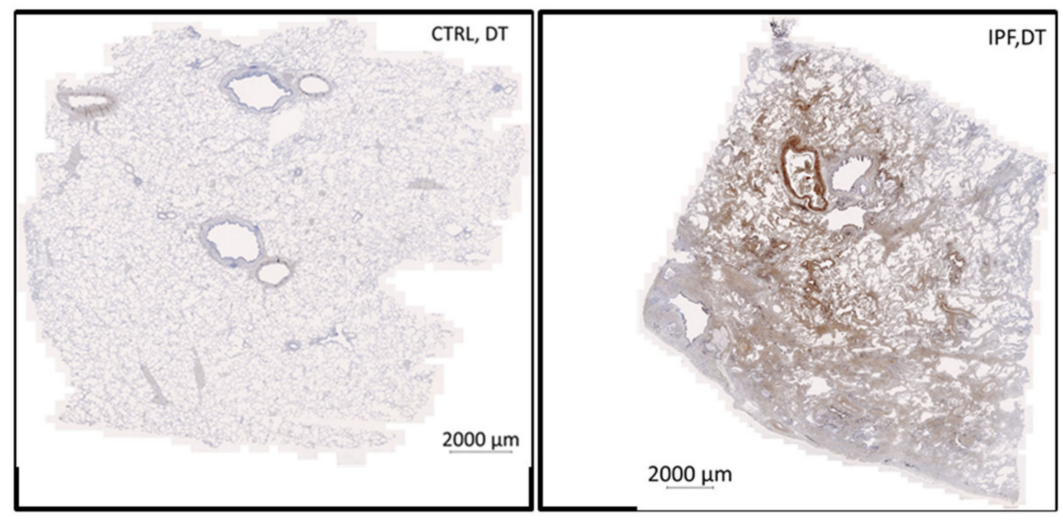

B

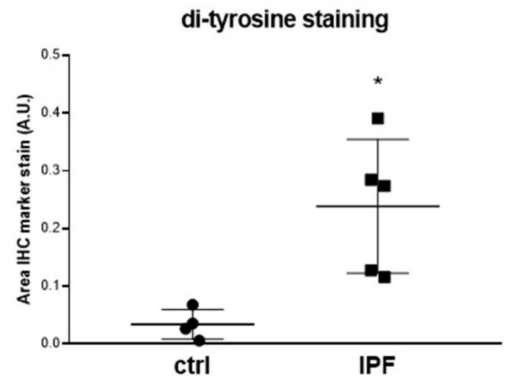

C

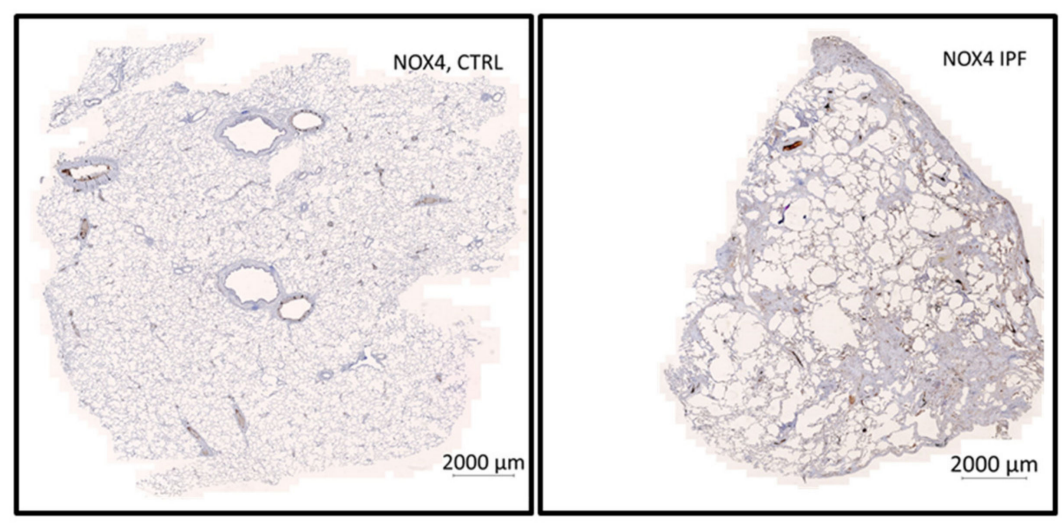

NOX4 staining

D

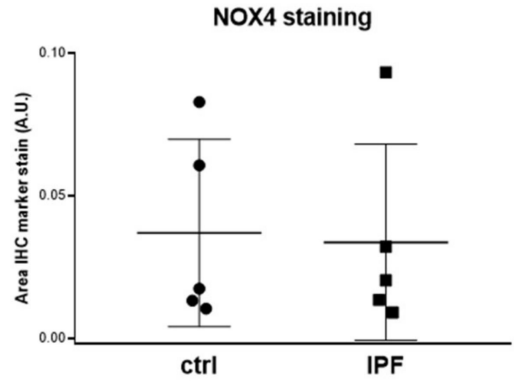

Figure 2. Representative images of DT (A) and NOX4 (C) staining in the indicated patients are shown for lungs. Quantification of staining for DT (B) and NOX4 (D). * $p<0.05$ using Mann-Whitney nonparametric test. CTRL: control; IPF: idiopathic pulmonary fibrosis.

We further analyzed the lung material used in this study and focused on the cell types stained by NOX4 and DT antibodies, namely smooth muscle cells, alveolar type II and bronchial epithelial cells and endothelial cells.

\subsection{DT and NOX4 Are Present in Thickened Vessel Walls in the Lungs of IPF Patients}

Vascular remodeling is a key pathological feature in the scarred areas of IPF lungs. It is characterized by the proliferation of pulmonary arterial smooth muscle cells in response 
to hypoxia leading to the thickening of the media, the smooth muscle layer around the blood vessels. Vascular smooth muscle cells in control lung tissues were negative for DT and NOX4 (Figure 3A). However, fibrotic regions of IPF lungs showed abnormal vascular architecture and a strong staining for both DT and NOX4 in pulmonary arterial smooth muscle cells (Figure 3B). At higher magnification, pulmonary arterial smooth muscle cells appeared highly positive for DT and to express high NOX4 levels (insets in Figure 3B). While DT staining appeared cytoplasmic, NOX4 staining was mainly found in perinuclear regions of smooth muscle cells. This indicates an upregulation of NOX4 and DT in a specific vascular pathological modification of IPF and may suggest a potential role of NOX4 in the generation of $\mathrm{H}_{2} \mathrm{O}_{2}$ needed for DT formation in proliferative pulmonary arterial smooth muscle cells.

A

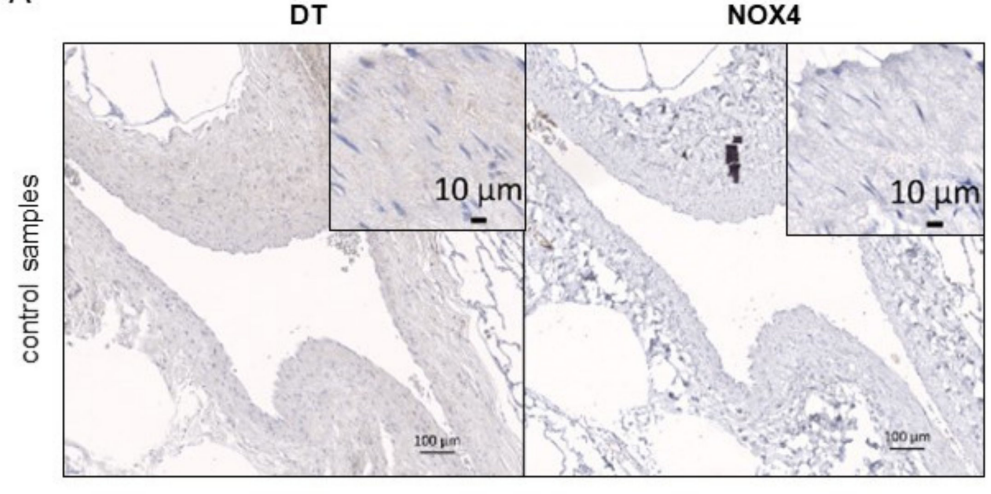

B DT NOX4

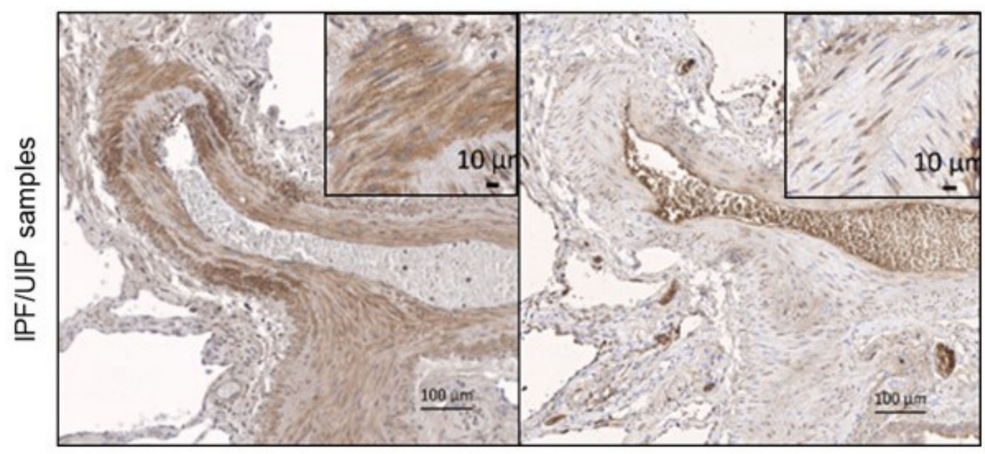

Figure 3. Representative images for DT ad NOX4 staining of blood vessel smooth muscle cells in the control (A) and IPF samples (B) of human patients are shown. Inset shows a $10 \times$ higher magnification and depicts typical smooth muscle cell staining in these samples. DT and NOX4 staining are shown in brown.

\subsection{DT Staining and NOX4 Expression in Bronchi and Alveolar Epithelial Cells Type II of IPF Patients}

The pulmonary alveolar epithelium is essential for the air-blood barrier function of the lungs. It is mainly composed of alveolar epithelial cells type I (AT1) and type II (AECII) cells. AT1 cells are abundant, large squamous cells involved in gas exchange, while AECII cells are smaller, cuboidal cells mostly involved in synthesis and secretion of the lung surfactant. Contrary to endothelial cells, smooth muscle cells, alveolar epithelial cells type II and macrophages which can be recognized by their tissue localization and morphology, it was impossible to correctly identify AT1 cells by IHC and hence we only looked at AECII cells. 
AECII were not stained with DT and NOX4 antibodies in control lung tissue. On the other hand, although the alveoli were severely damaged, both NOX4 and DT antibodies decorated the remaining AECII cells in IPF (Figure 4B), consistent with a possible role of NOX4 in DT formation in AECII.

A

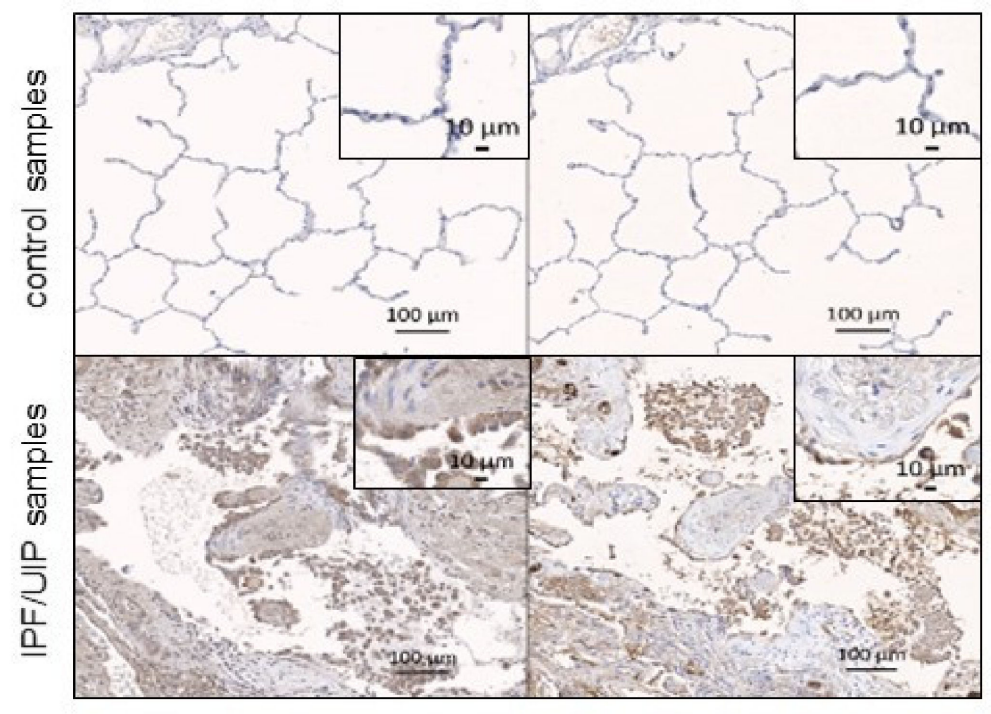

B

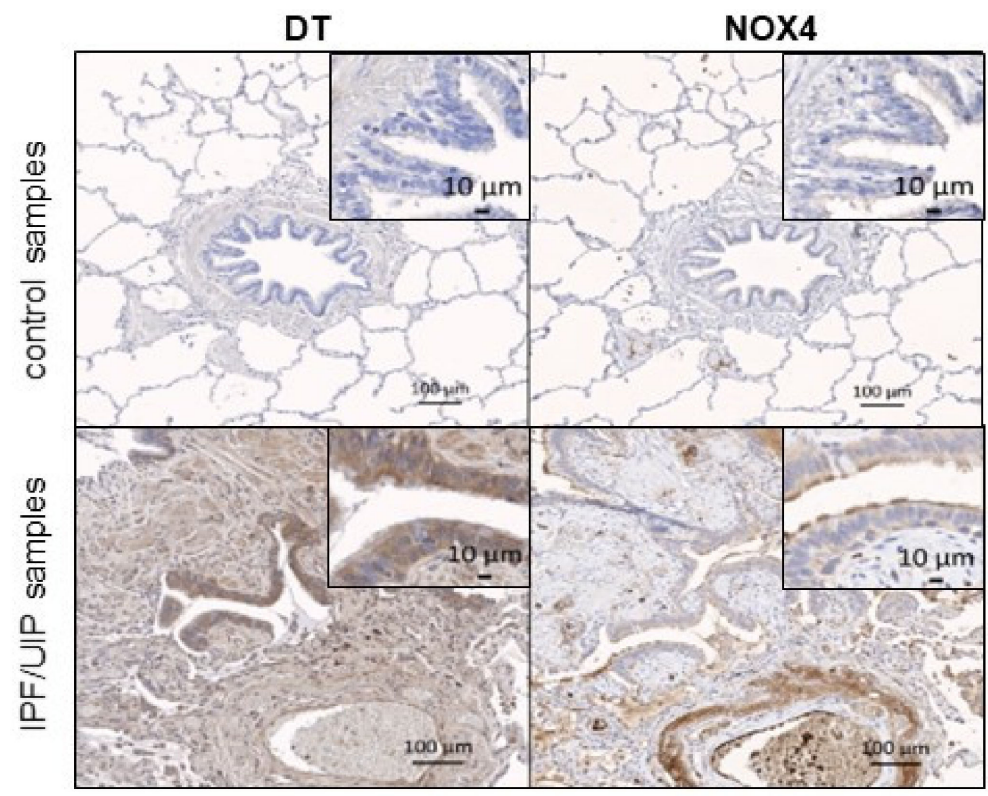

Figure 4. Representative images for DT and NOX4 staining of AECII cells (A) and bronchi (B) in the control (upper panel) and IPF/UIP samples (lower panel) of human patients are shown. Insets shows a $10 \times$ higher magnification and depicts AECII (A) and ciliary bronchial cells (B) in these samples. DT and NOX4 staining are shown in brown.

In normal AECII epithelial cells and bronchi, very little staining was observed for both markers (Figure 4A,B, upper panels). However, both AECII and bronchial cells were stained in IPF samples with NOX4 and DT antibody with a different intensity, depending on the patient (Figure $4 \mathrm{~A}, \mathrm{~B}$, lower panels). 


\subsection{Different Expression of DT and NOX4 in Vascular Endothelium in Normal and IPF Tissue}

Interestingly, while IPF tissue globally showed elevated levels of DT, only partial DT staining was observed in pulmonary endothelial cells (EC) (Figure 5). On the other hand, high NOX4 levels were present in the endothelial cells of large vessels in both control and IPF tissue. NOX4 staining was also detected in capillary endothelial cells in some samples (data not shown). Due to the high specificity of NOX4 staining in endothelial cells and the fact that NOX4 is generating $\mathrm{H}_{2} \mathrm{O}_{2}$ constitutively, the absence of DT staining in two out of four samples indicates that the conditions required for DT formation are not fulfilled in the intimal parts of the vessels.

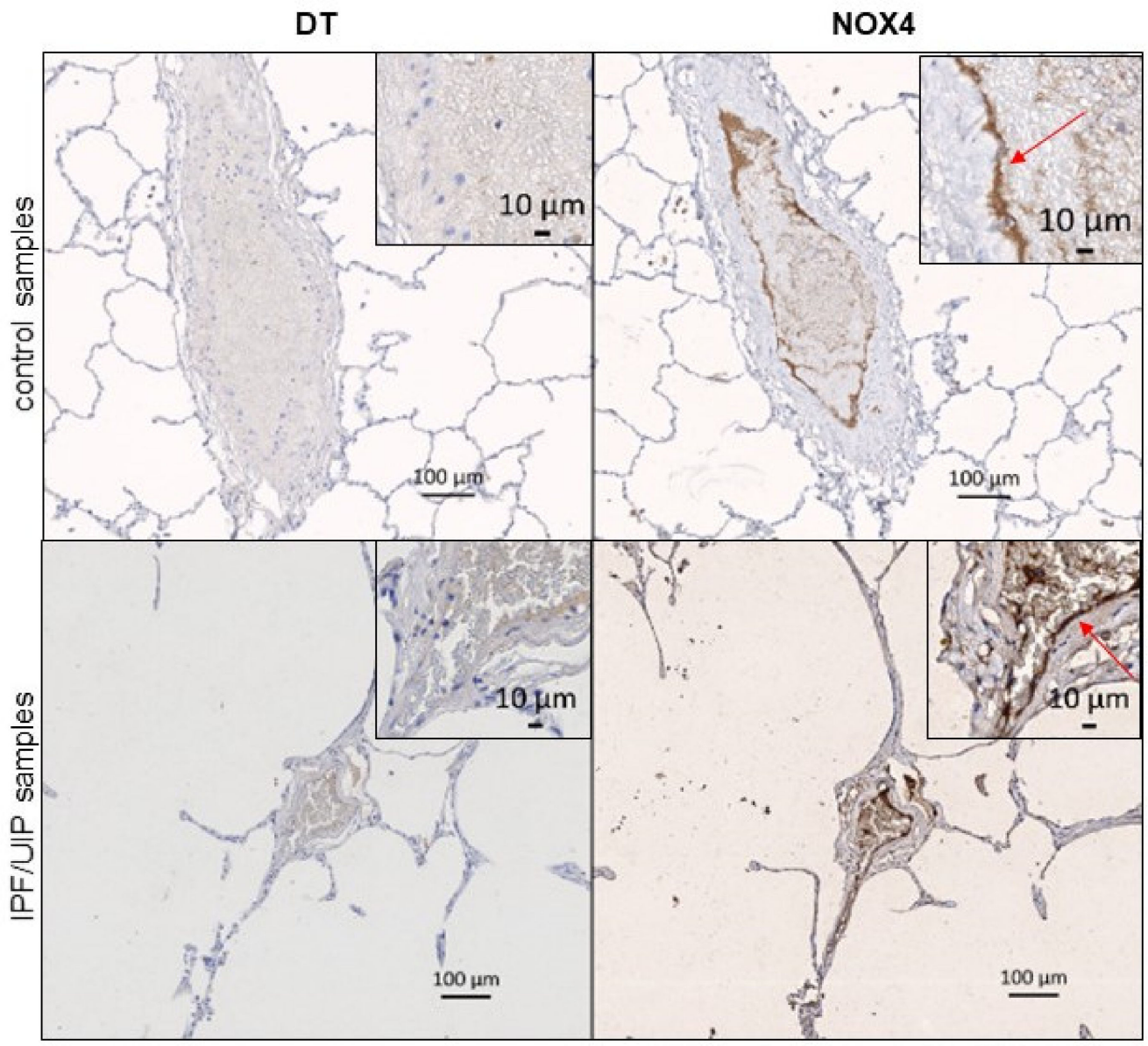

Figure 5. Representative images for DT and NOX4 staining of blood vessel endothelial cells in the control (upper panels) and the IPF/UIP samples (lower panels) from human patients are shown. Inset shows a 10X higher magnification and depicts typical intimal endothelial region of these samples. DT and NOX4 staining are shown in brown. The red arrows indicate the NOX4 endothelial staining.

Altogether, DT staining was found in none of our control samples, but was present in SMC, AEC and AECII in all tested IPF patients (Figure 6A). NOX4 staining was present in EC in both control and IPF samples, while AECII and SMC cells were mainly stained only in IPF patients (Figure 6B). Note that both antibodies stained macrophages in all samples (Figure 6A,B). 
A

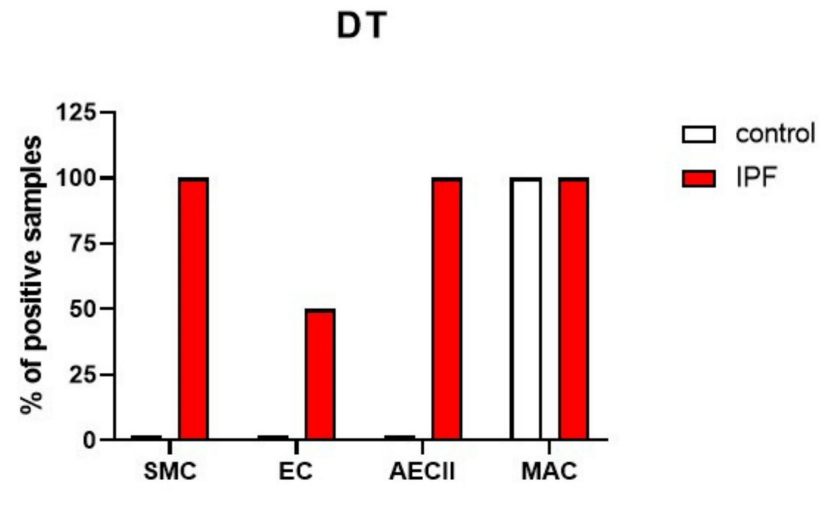

B

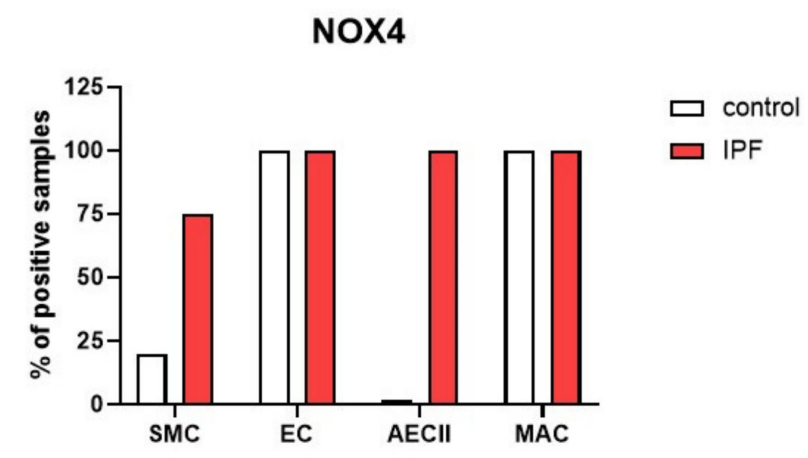

Figure 6. Histograms documenting the number of samples positive for DT and NOX4 for each cellular subtype. All 4 IPF patients were positive for DT in SMC and AECII while none of the controls showed DT staining (A). All cell types (SMC, AECII and EC were stained for NOX4 in IPF patients, while only EC were stained in the control (B). Macrophages were stained with both antibodies in both control and IPF (most likely not specific). SMC: smooth muscle cells, EC: endothelial cells, AECII: alveolar epithelial cells type 2, MAC macrophages.

\section{Discussion}

In this study, we showed that DT is a novel specific histopathological marker of IPF that can be detected with a specific antibody in lung tissue. We showed using differentiated myofibroblasts that NOX4 generated a sufficient amount of $\mathrm{H}_{2} \mathrm{O}_{2}$ for DT formation in vitro and that NOX4 and DT stained both proliferative pulmonary smooth muscle cells and epithelial bronchial and AECII cells. However, the endothelial cells of blood vessels were stained predominantly with NOX4 and independently of the disease, though some IPF samples also showed the staining with DT.

Our data confirm that DT accumulation in the lungs is a pathological feature of IPF. Indeed, our data confirmed previous reports showing increased levels of proteins containing DT modifications, in particular the tyrosine-rich ECM protein, fibronectin, in the plasma of patients with fibrotic interstitial lung disease and the bleomycin-induced IPF mouse model [15,16].

Since DT bridges are covalent and most likely irreversible in vivo, a pharmacological strategy using small chemical molecules or anti-DT antibodies is unlikely to be successful. However, the well-described mechanism leading to DT formation provides a rational for future therapies for IPF. Such an approach would aim at inhibiting one or several of the components necessary for DT formation, namely, (i) abundant levels of proteins of the ECM, (ii) one or several sources of $\mathrm{H}_{2} \mathrm{O}_{2}$ and (iii) the presence of an active hemecontaining peroxidase $[16,24,25,27]$. Existing therapeutic approaches in IPF that target 
the fundamental mechanisms of fibrosis leading to the deposition of ECM, including the proliferation and differentiation of fibroblasts are comprehensively reviewed in $[28,29]$ and will not be developed here.

More than 40 enzymes are described as generating $\mathrm{H}_{2} \mathrm{O}_{2}$ when they are active [8]. Among them, NADPH oxidases are highly relevant sources of $\mathrm{H}_{2} \mathrm{O}_{2}$ in the context of DT formation in IPF lungs. NOX is known to coordinate their activity with specific heme-containing peroxidases for key biological functions in humans and other organisms, including host defense and thyroid hormone synthesis (reviewed in [9]). Several NOX isoforms are expressed in the lungs, but most studies point towards a contribution of NOX4 in IPF. NOX4 is upregulated in IPF lungs while NOX4-deficient mice are partially protected in a bleomycin mouse model of IPF $[5,6]$. NOX4 expression is induced in fibroblasts concomitantly with ECM proteins following TGF $\beta 1$ treatment. Interestingly, high DT staining in dermal tissue observed during physiological wound healing is absent

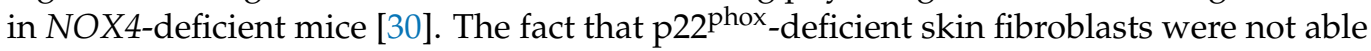
to generate DT crosslinking with TSA, in spite of the presence of high levels of ECM and an active heme peroxidase, supports a role of NOX4 in DT formation at least in the skin. No DT was observed in the sites with the highest NOX4 levels in endothelial cells in healthy tissue, but we detected some DT staining in two out of four IPF samples. One possibility is that in endothelial cells, NOX4 may generate $\mathrm{H}_{2} \mathrm{O}_{2}$ in the luminal side where ECM proteins or heme-containing peroxidases are absent. Alternatively, although the absence of colocalization does not exclude the diffusion of NOX4-derived $\mathrm{H}_{2} \mathrm{O}_{2}$ to other lung regions, NOX4-dependent DT formation might be limited to the fibrotic foci. Other sources of $\mathrm{H}_{2} \mathrm{O}_{2}$ might contribute to DT formation in IPF tissue. The dual oxidases DUOX1 and DUOX2 are expressed in airway epithelia where they generate $\mathrm{H}_{2} \mathrm{O}_{2}$ upon activation [31,32]. In particular DUOX1 and 2 may play a role in host defense in lung tissue through a coordinated function with the heme-containing lactoperoxidase [33]. DUOX1 was shown to be induced following lung injury, while DUOX1-deficient mice have an attenuated fibrotic phenotype in a bleomycin model of IPF [34]. However, to our knowledge, in spite of a strong rationale, the involvement of the DUOX1/lactoperoxidase coupling in DT formation in the lungs has not been studied yet.

\section{Conclusions}

In conclusion, our IHC study of postmortem and biopsy material describes elevated DT and specific DT and NOX4 expression patterns as typical features of IPF human pathology. The validation of a specific antibody against DT allowing the detection of a novel histopathological marker of IPF pathology grants a great impact in future studies aiming at dissecting the pathogenic roles played by DT formation in IPF. Our study opens a number of important questions for further investigation. including the timing of DT formation in IPF lungs, the types of ECM proteins undergoing DT formation, the alternative sources of $\mathrm{H}_{2} \mathrm{O}_{2}$ and the necessary heme-containing peroxidases leading to high DT in IPF. Exploration of these questions might lead to the discovery of novel therapeutics for IPF and potentially other fibrotic diseases.

Supplementary Materials: The following are available online at https://www.mdpi.com/article/10 .3390/antiox10111833/s1, Figure S1: NOX4 expression in human skin fibroblasts. Figure S2: Isotype antibody controls.

Author Contributions: Conceptualization, C.B.-A., S.B., I.R.-M. and K.-H.K.; methodology, S.B., Y.D., T.S., J.-C.P. and D.A.; formal analysis, S.B., J.-C.P. and V.J.; writing-original draft preparation, V.J. and S.B.; writing-review and editing, C.B.-A. and I.R.-M.; funding acquisition, C.B.-A. All authors have read and agreed to the published version of the manuscript.

Funding: This work was supported by grants to C. Barazzone-Argiroffo from the Swiss National Science Foundation (Grant 310030-159500/1) and to K.H. Krause (Grant 31003A-179478) and by the Swiss Lung Liga and the OPO-Stiftung, Switzerland. 
Institutional Review Board Statement: The study was conducted according to the guidelines of the Declaration of Helsinki, and approved by the regional research committee of the University of Geneva Hospital (Protocol no. 10-161R (NAC10-052R) and no. 11-087R (NAC 11-027R); date of approval 30 January 2011 and 10 June 2011).

Informed Consent Statement: Informed consent was obtained from all subjects involved in the study.

Data Availability Statement: Data is contained within the article or supplementary material.

Acknowledgments: The authors are grateful to the members of the Bioimaging Core Facility of the Faculty of Medicine, University of Geneva for their help in image analysis.

Conflicts of Interest: The authors declare no conflict of interest.

\section{References}

1. Wolters, P.J.; Collard, H.R.; Jones, K.D. Pathogenesis of Idiopathic Pulmonary Fibrosis. Annu. Rev. Pathol. Mech. Dis. 2014, 9, 157-179. [CrossRef]

2. Shumar, J.; Chandel, A.; King, C. Antifibrotic Therapies and Progressive Fibrosing Interstitial Lung Disease (PF-ILD): Building on INBUILD. J. Clin. Med. 2021, 10, 2285. [CrossRef]

3. Betensley, A.; Sharif, R.; Karamichos, D. A Systematic Review of the Role of Dysfunctional Wound Healing in the Patho-genesis and Treatment of Idiopathic Pulmonary Fibrosis. J. Clin. Med. 2016, 6, 2. [CrossRef] [PubMed]

4. Veith, C.; Boots, A.W.; Idris, M.; Van Schooten, F.-J.; Van Der Vliet, A. Redox Imbalance in Idiopathic Pulmonary Fibrosis: A Role for Oxidant Cross-Talk Between NADPH Oxidase Enzymes and Mitochondria. Antioxid. Redox Signal. 2019, 31, $1092-1115$. [CrossRef]

5. Hecker, L.; Vittal, R.; Jones, T.; Jagirdar, R.; Luckhardt, T.R.; Horowitz, J.; Pennathur, S.; Martinez, F.J.; Thannickal, V.J. NADPH oxidase-4 mediates myofibroblast activation and fibrogenic responses to lung injury. Nat. Med. 2009, 15, $1077-1081$. [CrossRef]

6. Carnesecchi, S.; Deffert, C.; Donati, Y.; Basset, O.; Hinz, B.; Preynat-Seauve, O.; Guichard, C.; Arbiser, J.L.; Banfi, B.; Pache, J.C.; et al. A key role for NOX4 in epithelial cell death during development of lung fibrosis. Antioxid. Redox Signal. 2011, 15, 607-619. [CrossRef] [PubMed]

7. Bedard, K.; Krause, K.H. The NOX Family of ROS-Generating NADPH Oxidases: Physiology and Pathophysiology. Physiol. Rev. 2007, 87, 245-313. [CrossRef]

8. Sies, H.; Jones, D.P. Reactive oxygen species (ROS) as pleiotropic physiological signalling agents. Nat. Rev. Mol. Cell Biol. 2020, 21, 363-383. [CrossRef] [PubMed]

9. Sirokmány, G.; Geiszt, M. The Relationship of NADPH Oxidases and Heme Peroxidases: Fallin' in and Out. Front. Immunol. 2019, 10, 394. [CrossRef]

10. Aeschbach, R.; Amadoò, R.; Neukom, H. Formation of dityrosine cross-links in proteins by oxidation of tyrosine residues. Biochim. Biophys. Acta Protein Struct. 1976, 439, 292-301. [CrossRef]

11. Wong, J.L.; Creton, R.; Wessel, G.M. The Oxidative Burst at Fertilization Is Dependent upon Activation of the Dual Oxidase Udx1. Dev. Cell 2004, 7, 801-814. [CrossRef]

12. Hensley, K.; Maidt, M.L.; Yu, Z.; Sang, H.; Markesbery, W.R.; Floyd, R.A. Electrochemical analysis of protein nitrotyrosine and dityrosine in the Alzheimer brain indicates region-specific accumulation. J. Neurosci. 1998, 18, 8126-8132. [CrossRef]

13. Al-Hilaly, Y.K.; Biasetti, L.; Blakeman, B.J.; Pollack, S.J.; Zibaee, S.; Abdul-Sada, A.; Thorpe, J.R.; Xue, W.F.; Serpell, L.C. The involvement of dityrosine crosslinking in alpha-synuclein assembly and deposition in Lewy Bodies in Parkinson's disease. Sci. Rep. 2016, 6, 39171. [CrossRef] [PubMed]

14. Leeuwenburgh, C.; Rasmussen, J.E.; Hsu, F.F.; Mueller, D.M.; Pennathur, S.; Heinecke, J.W. Mass Spectrometric Quantification of Markers for Protein Oxidation by Tyrosyl Radical, Copper, and Hydroxyl Radical in Low Density Lipoprotein Isolated from Human Atherosclerotic Plaques. J. Biol. Chem. 1997, 272, 3520-3526. [CrossRef] [PubMed]

15. Pennathur, S.; Vivekanandan-Giri, A.; Locy, M.L.; Kulkarni, T.; Zhi, D.; Zeng, L.; Byun, J.; de Andrade, J.A.; Thannickal, V.J. Oxidative Modifications of Protein Tyrosyl Residues Are Increased in Plasma of Human Subjects with In-terstitial Lung Disease. Am. J. Respir. Crit. Care Med. 2016, 193, 861-868. [CrossRef] [PubMed]

16. Locy, M.L.; Rangarajan, S.; Yang, S.; Johnson, M.R.; Bernard, K.; Kurundkar, A.; Bone, N.B.; Zmijewski, J.W.; Byun, J.; Pennathur, S.; et al. Oxidative cross-linking of fibronectin confers protease resistance and inhibits cellular migration. Sci. Signal. 2020, 13, eaau2803. [CrossRef] [PubMed]

17. Wells, R.G. Tissue mechanics and fibrosis. Biochim. Biophys. Acta Mol. Basis Dis. 2013, 1832, 884-890. [CrossRef]

18. Brault, J.; Goutagny, E.; Telugu, N.; Shao, K.; Baquié, M.; Satre, V.; Coutton, C.; Grunwald, D.; Brion, J.-P.; Barlogis, V.; et al. Optimized Generation of Functional Neutrophils and Macrophages from Patient-Specific Induced Pluripotent Stem Cells:Ex VivoModels of X0-Linked, AR220- and AR470- Chronic Granulomatous Diseases. BioRes. Open Access 2014, 3, 311-326. [CrossRef]

19. Prior, K.-K.; Leisegang, M.S.; Josipovic, I.; Löwe, O.; Shah, A.M.; Weissmann, N.; Schröder, K.; Brandes, R.P. CRISPR/Cas9mediated knockout of p22phox leads to loss of Nox1 and Nox4, but not Nox5 activity. Redox Biol. 2016, 9, 287-295. [CrossRef] [PubMed] 
20. Vandesompele, J.; De Preter, K.; Pattyn, F.; Poppe, B.; Van Roy, N.; De Paepe, A.; Speleman, F. Accurate normalization of real-time quantitative RT-PCR data by geometric averaging of multiple in-ternal control genes. Genome Biol. 2002, 3, RESEARCH0034. [CrossRef] [PubMed]

21. Weinberg, R.A.; Comb, M.J. Chapter 12: Immunohistochemistry (IHC) in CST Guide: Pathways E Protocols, 1st ed.; Cell Signaling Technology, Inc.: Danvers, MA, USA, 2015; pp. 214-217.

22. Diebold, B.A.; Wilder, S.G.; De Deken, X.; Meitzler, J.L.; Doroshow, J.H.; McCoy, J.W.; Zhu, Y.; Lambeth, J.D. Guidelines for the Detection of NADPH Oxidases by Immunoblot and RT-qPCR. Methods Mol. Biol. 2019, 1982, 191-229.

23. Augsburger, F.; Filippova, A.; Rasti, D.; Seredenina, T.; Lam, M.; Maghzal, G.; Mahiout, Z.; Jansen-Durr, P.; Knaus, U.G.; Doroshow, J.; et al. Pharmacological characterization of the seven human NOX isoforms and their inhibitors. Redox Biol. 2019, 26, 101272. [CrossRef]

24. Rajaram, R.D.; Dissard, R.; Faivre, A.; Ino, F.; Delitsikou, V.; Jaquet, V.; Cagarelli, T.; Lindenmeyer, M.; Jansen-Duerr, P.; Cohen, C.; et al. Tubular NOX4 expression decreases in chronic kidney disease but does not modify fibrosis evolution. Redox Biol. 2019, 26, 101234. [CrossRef]

25. Kato, Y.; Wub, X.; Naitoc, M.; Nomurac, H.; Kitamotoa, N.; Osawa, T. Immunochemical Detection of Protein Dityrosine in Atherosclerotic Lesion of Apo-E-Deficient Mice Using a Novel Monoclonal Antibody. Biochem. Biophys. Res. Commun. 2000, 275, 11-15. [CrossRef]

26. Larios, J.M.; Budhiraja, R.; Fanburg, B.L.; Thannickal, V.J. Oxidative protein cross-linking reactions involving L-tyrosine in transforming growth factor-beta1-stimulated fibroblasts. J. Biol. Chem. 2001, 276, 17437-17441. [CrossRef]

27. Woods, A.A.; Linton, S.M.; Davies, M. Detection of HOCl-mediated protein oxidation products in the extracellular matrix of human atherosclerotic plaques. Biochem. J. 2003, 370, 729-735. [CrossRef] [PubMed]

28. Maher, T.M.; Strek, M.E. Antifibrotic therapy for idiopathic pulmonary fibrosis: Time to treat. Respir. Res. 2019, 20, 1-9. [CrossRef]

29. Mora, A.L.; Rojas, M.; Pardo, A.; Selman, M. Emerging therapies for idiopathic pulmonary fibrosis, a progressive age-related disease. Nat. Rev. Drug Discov. 2017, 16, 755-772. [CrossRef] [PubMed]

30. Lévigne, D.; Modarressi, A.; Krause, K.-H.; Pittet-Cuénod, B. NADPH oxidase 4 deficiency leads to impaired wound repair and reduced dityrosine-crosslinking, but does not affect myofibroblast formation. Free. Radic. Biol. Med. 2016, 96, 374-384. [CrossRef]

31. Luxen, S.; Noack, D.; Frausto, M.; Davanture, S.; Torbett, B.E.; Knaus, U.G. Heterodimerization controls localization of DuoxDuoxA NADPH oxidases in airway cells. J. Cell Sci. 2009, 122, 1238-1247. [CrossRef] [PubMed]

32. Forteza, R.; Salathe, M.; Miot, F.; Forteza, R.; Conner, G.E. Regulated hydrogen peroxide production by Duox in human airway epithelial cells. Am. J. Respir. Cell Mol. Biol. 2005, 32, 462-469. [CrossRef] [PubMed]

33. Sarr, D.; Tóth, E.; Gingerich, A.; Rada, B. Antimicrobial actions of dual oxidases and lactoperoxidase. J. Microbiol. 2018, 56, 373-386. [CrossRef] [PubMed]

34. Louzada, R.A.; Corre, R.; Ameziane El Hassani, R.; Meziani, L.; Jaillet, M.; Cazes, A.; Crestani, B.; Deutsch, E.; Dupuy, C. NADPH oxidase DUOX1 sustains TGF-beta1 signalling and promotes lung fibrosis. Eur. Respir. J. 2021, 57, 1901949. [CrossRef] [PubMed] 